Published in final edited form as:

Science. 2019 December 06; 366(6470): . doi:10.1126/science.aax4380.

\title{
A generalized HIV vaccine design strategy for priming of broadly neutralizing antibody responses
}

\author{
Jon M. Steichen ${ }^{1,2,3, \dagger}$, Ying-Cing Lin ${ }^{4, \dagger}$, Colin Havenar-Daughton ${ }^{3,5, \dagger}$, Simone Pecetta $^{4, \dagger}$, \\ Gabriel Ozorowski ${ }^{2,3,6, \dagger}$, Jordan R. Willis ${ }^{1,2,3, \dagger}$, Laura Toy ${ }^{3,5}$, Devin Sok ${ }^{1,2,3}$, Alessia \\ Liguori $^{1,2,3}$, Sven Kratochvil ${ }^{4}$, Jonathan L. Torres ${ }^{2,3,6}$, Oleksandr Kalyuzhniy ${ }^{1,2,3}$, Eleonora \\ Melzi $^{4}$, Daniel W. Kulp ${ }^{1,2,3,7}$, Sebastian Raemisch ${ }^{1,2,3}$, Xiaozhen $\mathrm{Hu}^{1,2,3}$, Steffen M. \\ Bernard $^{2,3,6}$, Erik Georgeson ${ }^{1,2,3}$, Nicole Phelps ${ }^{1,2,3}$, Yumiko Adachi ${ }^{1,2,3}$, Michael \\ Kubitz $^{1,2,3}$, Elise Landais ${ }^{1,2,3}$, Jeffrey Umotoy 1,2,3, Amanda Robinson ${ }^{1,2,3}$, Bryan \\ Briney $^{1,2,3,8}$, lan A. Wilson ${ }^{2,3,6,9}$, Dennis R. Burton ${ }^{1,2,3}$, Andrew B. Ward ${ }^{2,3,6}$, Shane \\ Crotty ${ }^{3,5,10,{ }^{*}}$, Facundo D. Batista ${ }^{3,4,{ }^{*}}$, William R. Schief ${ }^{1,2,3,4,{ }^{*}}$ \\ ${ }^{1}$ Department of Immunology and Microbiology, The Scripps Research Institute, La Jolla, CA \\ 92037, USA.
}

${ }^{2}$ IAVI Neutralizing Antibody Center, The Scripps Research Institute, La Jolla, CA 92037, USA.

${ }^{3}$ Consortium for HIV/AIDS Vaccine Development, The Scripps Research Institute, La Jolla, CA 92037, USA.

${ }^{4}$ The Ragon Institute of Massachusetts General Hospital, Massachusetts Institute of Technology and Harvard University, Cambridge, MA 02139, USA.

${ }^{5}$ Division of Vaccine Discovery, La Jolla Institute for Immunology, La Jolla, CA 92037, USA.

${ }^{6}$ Department of Integrative Structural and Computational Biology, The Scripps Research Institute, La Jolla, CA 92037, USA.

${ }^{7}$ Vaccine and Immune Therapy Center, The Wistar Institute, Philadelphia, PA 19104, USA.

${ }^{8}$ Center for Viral Systems Biology, The Scripps Research Institute, La Jolla, CA 92037, USA

${ }^{9}$ Skaggs Institute for Chemical Biology, The Scripps Research Institute, La Jolla, CA 92037, USA

\footnotetext{
*Correspondence to: schief@scripps.edu, fbatista1@mgh.harvard.edu, shane@1ji.org.

Author Contributions: J.M.S. and W.R.S. conceived the study. J.M.S., Y-C.L., C.H-D., S.P., G.O., D.R.B., A.B.W., S.C., F.D.B., and W.R.S. designed the study. J.M.S., D.W.K., S.R., A.R., and W.R.S. designed immunogens. J.M.S. designed Abs. Y-C.L., S.P., S.K., E.M., and F.D.B performed immunization studies. C.H-D. and L.T. performed naïve B cell sorting studies. G.O. and J.L.T. performed cryo-EM studies. B.B. performed NGS. J.R.W. performed bioinformatics analyses. D.S., E.L., and J.U. performed neutralization assays. A.L., O.K., X.H. characterized immunogens and Abs. E.G., N.P., Y.A., M.K. purified proteins. S.M.B. and I.A.W. contributed structural information. J.M.S. and W.R.S. wrote the manuscript. All co-authors edited the manuscript.

$\dagger$ Contributed equally

Competing interests: S.P. is now employed by GSK Vaccines S.r.1., a company that might benefit indirectly from this research. D.R.B. is a paid consultant of IAVI. J.M.S. and W.R.S. are inventors on a patent application submitted by IAVI and The Scripps Research Institute that covers the N332-GT immunogens developed in this manuscript.

Data and materials availability: Coordinates and maps for the structural data presented in this manuscript have been deposited to the Protein Data Bank under accession codes 6DFG, 6DFH, 6NF5, 6NFC and 6OC7, and to the Electron Microscopy Data Bank under accession codes EMD-7875, EMD-7876, EMD-7884 and EMD-7885. Antibody sequences discovered during this study have been deposited to Genbank under accession numbers MN495018 to MN495471 (BG18gH mouse antibodies) and MN514889 to MN514945 (human naive B cell antibodies binding N332-GT immunogens). Custom scripts for NGS database query will be made available from the corresponding author upon request. All other data is available in the main text or supplementary materials.
} 
${ }^{10}$ Division of Infectious Diseases, Department of Medicine, University of California, San Diego, La Jolla, CA 92037, USA

\section{Abstract}

Vaccine induction of broadly neutralizing antibodies (bnAbs) to human immunodeficiency virus (HIV) remains a major challenge. Germline-targeting immunogens hold promise for initiating the induction of certain bnAb classes; yet for most bnAbs, a strong dependence on antibody heavy chain complementarity determining region 3 (HCDR3) is a major barrier. Exploiting ultra-deep human antibody sequencing data, we identified a diverse set of potential antibody precursors for a bnAb with dominant HCDR3 contacts. We then developed HIV envelope trimer-based immunogens that primed responses from rare bnAb-precursor B cells in a mouse model, and in exvivo screens bound a range of potential bnAb-precursor human naive B cells. Our repertoireguided germline-targeting approach provides a framework for priming the induction of many HIV bnAbs, and could be applied to most HCDR3-dominant antibodies from other pathogens.

\section{One Sentence Summary:}

Proof of principle for a method to design vaccine immunogens to prime the induction of antibodies to HIV and other pathogens.

HIV infects 1.8 million new people each year, making development of an HIV vaccine a global health priority (1). Nearly all licensed vaccines protect by inducing antibodies, but highly antigenically variable pathogens such as HIV and influenza have eluded traditional vaccine strategies $(2,3)$. The discoveries of broadly neutralizing antibodies (bnAbs) that bind to relatively conserved epitopes on viral surface proteins have inspired new vaccine design strategies $(4,5)$.

Antibodies, produced by B cells, acquire affinity-enhancing mutations when a B cell mutates and matures from the original naive B cell (or "germline") state. Germline-targeting HIV vaccine design aims to induce bnAbs by first priming bnAb-precursor B cells and then shepherding $\mathrm{B}$ cell affinity maturation with a series of rationally designed boosting immunogens. A key rationale for this strategy is that germline-reverted forms of bnAbsprecursors with all recognizable amino acid mutations reverted to germline-typically have no detectable affinity for HIV envelope (Env) proteins. Thus, for a vaccine to initiate bnAb induction, a germline-targeting priming immunogen with appreciable affinity for bn $\mathrm{Ab}$ precursors must be engineered. Most HIV bnAbs (and most antibodies to any pathogen) bind to their target by employing their heavy chain complementarity-determining region 3 (HCDR3) as a major binding determinant. Hence, an optimal HIV vaccine that induces multiple bnAbs to different HIV Env sites, and a general solution to germline-targeting vaccine design that could be applied broadly to other pathogens, will need to work with HCDR3-dependent antibodies. Many advances have been made in developing germlinetargeting immunogens to prime precursors for one particular class of bnAbs (VRC01-class bnAbs) (6-15), and at least one such immunogen has entered human clinical testing (16). However, VRC01-class bnAbs represent a specialized case in which non-HCDR3 features are the main determinants of antibody specificity and affinity (6-15). 
The need to design germline-targeting immunogens to initiate HCDR3-dependent bnAb responses brings new challenges. Although each B cell expresses a single unique antibody, different $\mathrm{B}$ cells produce diverse antibodies encoded by different combinations of antibody genes, with additional variation at junctions between genes, and the greatest antibody diversity is encoded in the HCDR3 portion of the molecule. The exceptional diversity in the human B cell repertoire makes any single bnAb-precursor HCDR3 sequence an impractical vaccine target. Rather, a pool of precursors sharing a set of bnAb-associated genetic features must be identified and targeted. Thus, owing to the antibody diversity in humans, a germline-targeting immunogen should have affinity for diverse bnAb precursors in order to succeed in diverse vaccine recipients.

\section{Strategy for Immunogen Design and Testing}

We report a potential solution to the above challenges. We selected the bnAb BG18 $(17,18)$ as a test case for a high value vaccine design target, because BG18 is the most potent bnAb directed to the N332-supersite, one of the major bnAb sites on HIV Env, and BG18 lacks insertions or deletions (indels) and therefore may be easier to induce than other bnAbs that require indels (see Supplementary text) (19). Using the strongly HCDR3-dependent bnAb BG18 $(17,18)$, we demonstrate a method to identify pools of bnAb potential precursors and use them as design targets to engineer HIV Env trimer immunogens that bind diverse bnAb potential precursors. We then provide pre-clinical validation by assessing these immunogens for: (i) their ability to select rare bnAb potential precursor naive B cells from the blood of HIV-seronegative human donors, (ii) their modes of binding to bnAb precursors, and (iii) their capacity to prime rare bnAb naive precursors with human physiological affinities in a mouse model (fig. S1).

\section{Precursor Frequency Analysis}

Crystal structures of BG18 bound to HIV Env trimers indicated a BG18 binding mode in which the HCDR3 engages the conserved GDIR motif at the base of the V3 loop like the bnAb PGT121, while the HCDR1 contacts the relatively conserved N332 glycan, and the light chain (LC) straddles the V1 loop of gp120, unlike PGT121 (18). This binding mode was corroborated by 1) structural modeling (fig. S2, A to D); 2) a 4.4 A resolution cryo-EM structure of BG18 bound to an HIV Env trimer (Figs 1A S3, and table S2); 3) mutagenesis studies (fig. S2, E to F); and 4) structural model-guided design of a minimally mutated BG18 bnAb (minBG18) that retained $\sim 67 \%$ of the neutralization breadth of BG18 with only $11 \%$ amino acid mutations in $\mathrm{V}_{\mathrm{H}} \mathrm{V}_{\mathrm{L}}$ compared to $30 \%$ for BG18 (fig. S4). The successful design of minBG18 provided an additional rationale for BG18 vaccine targeting, namely that the high mutation level in BG18 itself is not required to achieve substantial neutralization breadth and potency in a BG18-like response. The elucidation of the BG18 binding mode by these studies enabled structure-guided immunogen design.

To assess the extent to which BG18-like precursor HCDR3s are present in the general population, we used a bioinformatics approach to search a custom next-generation sequencing (NGS) dataset of $1.1 \times 10^{9}$ sequences of human B cell receptor (BCR) heavy chains (HCs) from 14 healthy, HIV seronegative donors $\left(8.58 \times 10^{8}\right.$ sequences from 4 donors 
were obtained in this work (20) and were combined with $2.55 \times 10^{8}$ sequences from 10 donors from ref. (21)). Informed by our structural model for the BG18-Env interaction, we searched for BG18-like HCDR3 sequences with the same length as BG18, the same D gene in the same reading frame and position within the HCDR3, and the same $\mathrm{J}_{\mathrm{H}}$ gene, allowing for diverse V-D and D-J junctions. Such BG18-like HCDR3 sequences were identified in all 14 donors (fig. S5), encouraging us to proceed with vaccine design. We further hypothesized that a range of BG18-like bnAbs utilizing alternate $\mathrm{V}_{\mathrm{H}}$ or $\mathrm{V}_{\mathrm{L}}$ genes could potentially interact with Env in a similar HCDR3-dependent binding mode. This hypothesis was subsequently supported by our ability to engineer BG18-like bnAbs utilizing three alternate $\mathrm{V}_{\mathrm{L}}$ genes $\left(\mathrm{V}_{\mathrm{L}} 3-1, \mathrm{~V}_{\mathrm{L}} 3-21\right.$ and $\left.\mathrm{V}_{\mathrm{L}} 2-8\right)$ and two alternate $\mathrm{V}_{\mathrm{H}}$ genes $\left(\mathrm{V}_{\mathrm{H}} 4-59\right.$ and $\left.\mathrm{V}_{\mathrm{H}} 4-61\right)$ (fig. S6). Identification of diverse BG18-like precursor HCDR3s from NGS data, and construction of BG18-like bnAbs with alternate $\mathrm{V}_{\mathrm{H}}$ or $\mathrm{V}_{\mathrm{L}}$ genes, led us to target a broad range of BG18-like precursors in the germline-targeting design process.

\section{Design and Antigenic Analysis of Immunogens}

Germline-targeting immunogen design was carried out using a directed evolution method for engineering trimers on the surface of mammalian cells $(22,23)$. We considered that it would be important to overcome the limitations of using only inferred-germline (iGL) antibodies (BG18 iGL $_{0-2}$, fig. S5B) for the directed evolution of a germline-targeting immunogen with strong HCDR3 contacts. Our reasoning was that germline-targeting design processes directed to only iGL antibodies may fail to produce immunogens with appreciable affinity for diverse naive precursors. iGL antibodies contain bnAb HCDR3 junctions that have been selected and most likely somatically mutated for high affinity Env binding during bnAb affinity maturation. Therefore, such iGL antibodies may have features not present in the naive human antibody sequence repertoire. Furthermore, iGL antibodies likely underrepresent the diversity of potential precursors. We therefore designed a set of 15 BG18-like precursor antibodies that use BG18 germline-reverted genes but contain naive human BG18like HCDR3s with diverse junction regions identified in our search of NGS data described above (fig. S5B). Based on our findings that BG18-like bnAbs can utilize alternate $\mathrm{V}_{\mathrm{H}}$ and $\mathrm{V}_{\mathrm{L}}$ genes, we produced 10 additional BG18-like precursor antibodies with alternate $\mathrm{V}_{\mathrm{H}}$ or $\mathrm{V}_{\mathrm{L}}$ (fig. S7). This gave us 28 potential BG18-like precursors that could be used as selection reagents for directed evolution and multitarget optimization of Env trimer immunogens capable of binding and priming a broad range of BG18-like precursor B cells.

Seven Env mammalian cell surface display libraries, encoding amino acid variation within and around the BG18 epitope, were screened iteratively (20). At each stage, selection antibodies were used to isolate the highest affinity clones from the library, and the best mutations were incorporated into the next generation Env immunogen. The first library was based on a previously described immunogen, 11 mut $_{B}(22)$, that had weak but detectable affinity for BG18 $\mathrm{iGL}_{2}$, the first selection antibody employed (Supplementary text, Fig. 1C, table S1, and fig. S8). In the early iterations, libraries were screened against the least challenging selection antibodies (e.g., BG18 iGL) while in later stages libraries were screened against more difficult antibody targets (e.g., NGS-derived and alternate $\mathrm{V}_{\mathrm{H}} / \mathrm{V}_{\mathrm{L}}$ precursors) (Fig. 1C, table S1, fig. S5, and fig. S7). This directed evolution design process resulted in a series of germline-targeting Env trimers with increasing affinity for BG18 
precursors (N332-GT1, -GT2, and -GT5; Fig. 1C-D, table S1, and fig. S8). N332-GT5 trimer bound with a $\mathrm{K}_{\mathrm{D}}$ of $\sim 2 \mathrm{pM}$ to $\mathrm{BG}_{18} \mathrm{iGL}_{1}$, which represented a $\sim 14$ million-fold improvement over the initial protein design, 11mutB. More importantly, whereas the 14 NGS-derived precursors tested had undetectable affinity to the initial protein design (and undetectable affinity for native HIV Env trimer MD39, Fig. 1D), the design process resulted in 11/14 acquiring affinity to N332-GT2 (geomean $\mathrm{K}_{\mathrm{D}}=519 \mathrm{nM}$, Fig. 1D) and 12/14 binding to N332-GT5 (geomean $\mathrm{K}_{\mathrm{D}}=234 \mathrm{nM}$, Fig 1D) (One of the 15 NGS-derived precursors was found to be highly polyreactive and was therefore not included in our SPR analyses). Additionally, while only $3 / 10$ alternate $\mathrm{V}_{\mathrm{H}} / \mathrm{V}_{\mathrm{L}}$ precursor antibodies bound the starting protein design with low affinity $\left(\mathrm{K}_{\mathrm{D}}>10 \mu \mathrm{M}\right)$, and none bound native HIV Env trimer, (Fig. 1D), all 10 bound to N332-GT2 and N332-GT5 trimers, with robust affinities (geomean $\mathrm{K}_{\mathrm{D}}=11 \mathrm{nM}$ and $572 \mathrm{pM}$, respectively, Fig 1D). A $K_{\mathrm{D}} \leq 1 \mu \mathrm{M}$ may represent an affinity benchmark for generating robust germinal center (GC) responses from rare B cell precursors in vivo (24), and 20/27 potential bnAb precursors bound to N332-GT5 Env trimer with affinities of $K_{\mathrm{D}} \leq 1 \mu \mathrm{M}$ (Fig. 1D). Thus, the design process succeeded in extensively improving the immunogen binding properties to potential bnAb precursors with diverse HCDR3s and a variety of $\mathrm{V}_{\mathrm{H}}$ and $\mathrm{V}_{\mathrm{L}}$ genes.

\section{Immunogen Structural Analysis}

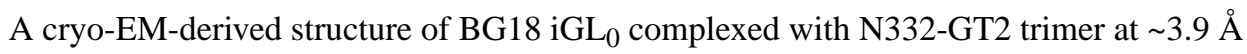
resolution (Fig. 1B and table S2) showed that $\mathrm{BG}_{18} \mathrm{iGL}_{0} \mathrm{HCDR} 3$ made a similar interaction to the base of the $\mathrm{V} 3$ as the HCDR3 of mature BG18 bound to the native-like trimer MD39 (Fig. 1A). Most of the additional interactions of BG18 iGL $_{0}$ complexed with N332-GT2 arise from V1 mutations in N332-GT2 that occupy a groove in the LC and also contact HCDR3 (fig. S9 and S10). HCDR3 dominates the interaction in the BG18 iGL $_{0}$ complex with N332-GT2, accounting for $64 \%$ of the total buried surface area. In the mature BG18 complex with MD39 Env trimer, HCDR3 maintains the same key interactions and contributes $35 \%$ of the total buried area as the antibody makes substantially increased contacts to glycans N332, N392, and N137 (table S3). Overall, cryo-EM structures showed that N332-GT2 binds to BG18 iGL $_{0}$ with a similar HCDR3-dependent, V1-straddling binding mode as BG505 MD39 Env trimer does with BG18.

\section{Immunogenicity Testing in a Mouse Model with Rare bnAb Precursors}

To test the immunogenicity of N332-GT2 Env trimer, we employed a BG1 ${ }^{\mathrm{gH}}$ knock-in mouse engineered via a CRISPR/Cas9 rapid targeting strategy, in which $\sim 30 \%$ of B cells express the $\mathrm{BG} 18 \mathrm{iGL}_{2} \mathrm{HC}$ variable region and mouse constant region paired with mouse LCs (25). The N332-GT2 Env trimer (but not MD39) bound to $12 \pm 1 \%$ of naive B cells in this mouse compared to $0.06 \pm 0.01 \%$ in WT (C57BL/6) mice, demonstrating N332-GT2 specificity for BG1 $8^{\mathrm{gH}}$ naive B cells (Fig. 2, A and B). Antigen-specific single B cell sorting and BCR sequencing demonstrated that the N332-GT2-specific naive BG1 ${ }^{\mathrm{gH}} \mathrm{B}$ cells carry a variety of mouse LCs paired with BG1 ${ }^{\mathrm{gH}}$ (Fig. 2C). Furthermore, N332-GT2 had similar affinities for naive BG1 $8^{\mathrm{gH}}$ B cell Fabs (geomean $K_{\mathrm{D}}$ of $582 \mathrm{nM}$ ) as for NGS-derived human BG18-like precursors (geomean $K_{\mathrm{D}}$ of $519 \mathrm{nM}$ ), showing the physiological relevance of the BG18-like precursor affinities in this mouse model. 
To generate a mouse model with rare bnAb precursor $\mathrm{B}$ cells, we carried out adoptive transfer experiments in which 5,000 CD45.2 BG1 $8^{\mathrm{gH}} \mathrm{B}$ cells were transferred to CD45.1 WT mice on day -1 , establishing a frequency of $\sim 7 \mathrm{GT}^{++} / \mathrm{KO}^{-} \mathrm{BG} 18^{\mathrm{gH}} \mathrm{CD} 45.2 \mathrm{~B}$ cells per million CD45.1 B cells by day 0 (fig. S11). Control transfers were 50,000 CD45.2 WT B cells. Previously, we constructed ferritin nanoparticles (NPs) that displayed up to 8 copies of MD39 native-like trimers (26), and mouse immunization studies showed that such NPs were superior to MD39 trimers in trafficking to follicular dendritic cell networks, concentrating in GCs, and eliciting IgG responses (27). We therefore engineered ferritin NPs displaying N332-GT2 trimers (fig. S12). Recipient mice were immunized at day 0 with either N332GT2-NPs or control NPs displaying MD39 trimers lacking GT mutations, for a total of four immunization conditions (BG18 ${ }^{\mathrm{gH}}$ or WT B cells transferred, N332-GT2- or MD39-NPs immunized). Splenocytes were analyzed by cytometry at day 8 (Fig. 2, D and E, and fig. S13). GC B cells $\left(\mathrm{CD} 38^{\text {low }} \mathrm{CD} 95^{+}\right)$were detected in all four immunization conditions, but CD45.2 GC B cells were detected only in the case of N332-GT2-NP immunization of BG1 ${ }^{\mathrm{gH}} \mathrm{B}$ cell recipients, demonstrating that $\mathrm{N} 332-\mathrm{GT} 2-\mathrm{NPs}$ activated rare BG18 ${ }^{\mathrm{gH}}$ B cells in vivo but MD39-NPs did not (Fig. 2D). N332-GT2-NPs induced CD45.2 GC B cells that bound to N332-GT2 and not to N332-GT2-KO (Fig. 2E) and were thus epitope-specific, consistent with a BG18-like response. In contrast, the same NPs induced considerably weaker epitope-specific responses among host CD45.1 GC B cells (Fig. 2E). In day 14 serum binding analyses, N332-GT2-NPs induced strong epitope-specific IgG responses in BG1 ${ }^{\mathrm{gH}} \mathrm{B}$ cell recipients and 15-fold weaker epitope-specific responses in WT B cell recipients (Fig. 2F), qualitatively consistent with the day $8 \mathrm{GC}$ data. This demonstrated that activation of rare $\mathrm{BG} 18^{\mathrm{gH}}$ precursor $\mathrm{B}$ cells led to potent serum antibody responses, and also showed that WT B cells responded to the BG18 epitope on N332-GT2. In contrast, MD39NPs induced negligible BG18-epitope-specific serum responses in either BG1 $8^{\mathrm{gH}}$ or WT B cell recipients (Fig. 2F). Together these results demonstrated that N332-GT2-NPs elicited $\mathrm{GC}$ and antibody responses from rare $\mathrm{BG} 18^{\mathrm{gH}} \mathrm{B}$ cells.

By single cell sorting and BCR sequencing CD $45.2^{+} / \mathrm{N} 332-\mathrm{GT}^{++} / \mathrm{KO}^{-} \mathrm{GC}$ B cells from BG1 $8^{\mathrm{gH}}$ recipient mice immunized with N332-GT2-NPs, we obtained HC-LC pairs at day 8 and day $42.100 \%$ of the $\mathrm{HCs}$ were derived from $\mathrm{BG} 18^{\mathrm{gH}}$, formally proving that these GC responses utilized the knockin $\mathrm{HC}$ (Fig. 2G). In contrast to the wide variety of mouse kappa genes used in LCs of N332-GT2-specific naive BG18 ${ }^{\mathrm{gH}} \mathrm{B}$ cells, by day 8 the LCs from GC B cells were highly enriched for two mouse kappa genes: Igkv12-46 and Igkv12-44 (Fig. $2 \mathrm{G})$. By day 42 , GC BCRs showed substantial SHM, diversification, and affinity maturation compared to naive B cells or day 8 GC BCRs (Fig. 2, H and I, and fig. S14). BG1 $8^{\mathrm{gH}} \mathrm{BCR}$ Fab affinities for N332-GT2 trimers increased by a factor of $\sim 6$ from day 0 to day 8 (geomean $\mathrm{K}_{\mathrm{D}}$ of $582 \mathrm{nM}$ and $97 \mathrm{nM}$, respectively, Fig. 2H). BG1 $8^{\mathrm{gH}}$ BCR Fab affinities increased dramatically by a factor of $\sim 900$ from day 0 to day 42 (geomean $K_{D}=640 \mathrm{pM}$, Fig. 2H). We conclude that N332-GT NPs can induce sustained GC responses and considerable affinity maturation and diversification from rare BG18-like precursors with human physiological affinities (see below), even in the presence of polyclonal competition.

To assess whether the affinity maturation induced by this single priming immunization was on a potential path toward bnAb development, we tested whether day 42 antibodies could bind Env trimers more native-like than the germline-targeting immunogen or neutralize 
viruses with more native-like Env. The N332-GT2 immunogen has 17 germline-targeting mutations, 8 of which are in two highly conserved regions of HIV Env (base of V3 loop around the GDIR motif, and $\beta 19$ ) and 9 of which are in one highly variable region (V1 loop) (fig. S8). Thus, a key question was whether antibodies induced by N332-GT2 could recognize Env trimers with more native sequences lacking mutations in the two conserved regions. We therefore constructed a BG505 Env stabilized trimer that included 8 of the N332-GT2 mutations in the V1 loop but was otherwise native-like (BG505-V1 mod) (fig. S15 and table S1), and we tested nine of the day 42 Fabs (those with highest affinity for N332GT2) for their ability to bind this V1-modified Env trimer in SPR. All nine Fabs bound BG505-V1 $1_{\text {mod }}$, with a geomean $\mathrm{K}_{\mathrm{D}}$ of $49 \mathrm{nM}$ (Fig. 2J). In contrast, five naive Fabs (those with highest affinity for N332-GT2) bound BG505-V1 mod 200 -fold more weakly, with a geomean $\mathrm{K}_{\mathrm{D}}$ of $10 \mu \mathrm{M}$, and inferred-germline variants of the day 42 Fabs (day 42 iGL Fabs) either showed no detectable affinity (4/5 tested) or bound weakly $(10 \mu \mathrm{M})$ (Fig. 2J). Furthermore, three of the day 42 Fabs, but none of the day 42 iGL Fabs, bound to the BG505 "7mut" trimer that is only six mutations away from a native-like Env trimer and was previously shown to be on-pathway to the development of PGT121-class bnAbs $(22,28)$ (Fig. 2J, fig. S15 and table S1). The day 42 antibodies did not bind N332-GT2-KO, consistent with BG18-like binding (Fig. 2J). None of the day 42 Fabs had detectable binding to the native-like trimer BG505 MD39, which was not surprising given the 17 mutation difference between the N332-GT2 immunogen and MD39 (Fig. 2J and fig. S8).

Neutralization assays with BG505 wild-type and $\mathrm{V} 1_{\text {mod }}$ HIV pseudoviruses were consistent with our SPR findings: five of six day 42 Fabs tested neutralized $V 1_{\text {mod }}$ HIV but not wildtype HIV, and none of the naive or day 42 iGL Fabs neutralized either virus (Fig. 2K). We conclude that a single N332-GT2 NP priming immunization elicited functional BG18-like antibodies that could bind and neutralize viruses bearing Env that retains HIV-conserved regions and is more native-like than the immunogen.

To assess whether the affinity maturation due to priming conferred a degree of reactivity breadth beyond clade A BG505, we tested whether day 42 Fabs could bind to HIV Env trimers from three different isolates and two additional clades (SF162P3 and AC10, clade B; AD8, clade C), all with the same modified V1 loop as BG505-V1 mod (fig. S15 and table S1). All nine day 42 Fabs tested bound to the three Env trimers with highly heterologous sequences, with geomean $\mathrm{K}_{\mathrm{D}} \mathrm{s}$ of 50,110 , and $69 \mathrm{nM}$ for SF162-V1 $1_{\text {mod }}$, AC10-V1 mod and AD8-V1 $1_{\text {mod }}$, respectively. In contrast, $4 / 5$ day 42 iGL Fabs had no detectable affinity for these trimers (Fig. 2J). These data show that priming with N332-GT2 in this mouse model induced antibodies with a substantial degree of breadth in that they can bind with relatively high affinity to diverse stabilized Env trimers only sharing the same V1 loop.

\section{Immunogen Reactivity with Naive Human B Cells}

A critical test of the germline-targeting design process was to determine if the N332-GT Env trimers could bind rare bnAb precursor human naive B cells (29). To our knowledge, this is a human immunogen design benchmark that has been met previously only by the germlinetargeting immunogen eOD-GT8 that targets VRC01-class bnAb precursors $(9,15)$. Attempts to isolate PGT121-related bnAb precursors using 11mut related trimers did not succeed (Supplementary Text, fig. S16), consistent with our hypothesis that germline-targeting 
design using only iGL antibodies would be unsuccessful because of an inability to accommodate the natural sequence diversity among bnAb precursors in human $\mathrm{B}$ cell repertoires. To probe human naive B cell reactivity to N332-GT Env trimers, we used N332GT1 and N332-GT2 as sorting reagents and either BG505-MD39 Env (containing a native N332 epitope) or N332-GT2-KO Env (an epitope knockout) as negative sorting probes (Fig. 3A). Approximately 16 million naive B cells from 6 donors were probed with N332-GT1, and 62 million naive B cells from 10 donors with N332-GT2, after accounting for PCR and sorting efficiencies (table S4). All donors for ex vivo B cell sorting were distinct from the 14 NGS donors mentioned above (20). N332 glycan supersite epitope-specific naive B cells (termed high mannose patch clones here, HMP) were isolated at a frequency of $\sim 0.001 \%$ (Fig. 3B and fig. S17). These epitope-specific B cells were enriched for long HCDR3s (Fig. 3C). The $B$ cells were also highly enriched for $V_{L} 3-25$ and $V_{L} 3-1$ LCs (Fig. 3D), which corresponded to the $\mathrm{BG} 18 \mathrm{~V}_{\mathrm{L}}$ and a $\mathrm{V}_{\mathrm{L}}$ that we showed could be used by BG18-like precursors and bnAbs (Fig. 1D and fig. S6). We expressed and purified Fabs from 46 HMP naive B cell clones (table S5) for further examination of the biochemical properties and specificities of the naive Abs. 23 HMP Fabs bound to N332-GT1 and/or GT2 by SPR and did not bind detectably to the MD39 native-like trimer, demonstrating proper N332 glycan supersite epitope specificity (Fig. 3, E and F). These SPR-validated epitope-specific clones were highly enriched for $V_{L} 3-25, V_{L} 3-1$, or the closely related $V_{L} 3-10$ (Fig. 3E). Thus, the protein design strategy resulted in Env trimers that could successfully bind human naive $\mathrm{B}$ cells with BG18-like LCs.

All N332-supersite bnAbs identified to date require a long HCDR3 ( $\geq 20 \mathrm{AA}$ ), due to the structural requirements for the bnAb HCDR3 to reach the HIV Env protein surface at the base of V3 while avoiding V1 loop glycans (19). While epitope-specific human B cells with HCDR3 lengths <20 AA were isolated using N332-GT1/2, only 4 of 8 such clones tested by SPR were confirmed epitope-specific, and their binding was weak $(>10 \mu \mathrm{M})$ (Fig. 3F and table S6). We considered that such B cells with HCDR3s <20 AA are probably unable to develop into N332-supersite bnAbs, and thus we did not study those clones further. Numerous epitope-specific naive B cell clones with HCDR3s $\geq 20$ AA were isolated with N332-GT1/2 probes (Fig. 4, A and B). From these human naive B cell clones, we identified two categories of potential BG18-like precursors. The first category shared the same HCDR3 length, D gene, D gene reading frame, D gene position within HCDR3, and $\mathrm{J}_{\mathrm{H}}$ gene with BG18 (Fig. 4A), exactly matching our initial search criteria when scanning NGS data for BG18-like HCDR3 sequences. Such naive B cells were termed type I BG18-like precursors. The second category of epitope-specific BG18-like B cells had VL3-25/VL3-1/ VL3-10 and long HCDR3s ( 220 AA) with diverse HC sequences (Fig. 4B). We termed this more diverse class of isolated naive B cells type II BG18-like precursors. HMP1 was a type I BG18-like precursor (Fig. 4A) with high affinity for N332-GT2 Env trimer $\left(\mathrm{K}_{\mathrm{D}}=220 \mathrm{nM}\right.$,

Fig. 3F). The type II BG18-like precursors with confirmed binding exhibited a geomean $\mathrm{K}_{\mathrm{D}}$ of $10 \mu \mathrm{M}$ for N332-GT2 Env trimer (Fig. 3F). Overall, type I and type II precursors accounted for 74\% (17/23) of the HMP Fabs isolated by N332-GT1 or N332-GT2 and verified as epitope-specific by SPR (Fig. 3, E and F), indicating that such BG18-like precursors may represent a substantial fraction of the human naive epitope-specific repertoire to these Env trimers. We then isolated additional type I and type II naive B cell 
clones using N332-GT5 Env trimer probes with additional blood donors (Fig. 4, A and B). Overall, three type I BG18-like precursors were isolated at a frequency of $\sim 1$ in 53 million naive B cells (HMP1, HMP68, and HMP69; table S4), in good agreement with our initial NGS bioinformatics-based estimate that precursors with BG18-like HCDR3s specific for N332-GT trimers may be present in the human B cell repertoire at a frequency of 1 in 54 million naive B cells (fig. S5). Type II BG18-like precursors were isolated at a higher frequency of $\sim 1$ in 7 million naive B cells, consistent with their larger sequence space.

\section{Structural Analysis of BG18-like Human Precursors Bound to Immunogens}

To gain a structural understanding for the potential of human type I and type II BG18-like precursors (Fig. 4, A and B) to mature into bnAbs, we solved high resolution cryo-EM structures of the complexes of N332-GT5 bound to HMP1 (type I) and HMP42 (type II), with resolutions of $3.7 \AA$ and $3.4 \AA$, respectively (Fig. 4, C and D, and table S2). Both HMPs showed a similar LC binding mode as BG18 iGL, with the LC straddling the V1 loop (Fig. 4C and fig. S10). The HCDR3 of HMP1 and BG18 iGL have nearly identical conformations, supporting HMP1 and type I class naive antibodies as ideal BG18 precursors (Fig. 4D). The projecting HCDR3 tip of HMP42 interacts with the same Env patch as BG18, but has a slightly different overall conformation that makes additional contacts with the Env V1 loop (Fig. 4D). This structural information supports the hypothesis that some or possibly all type II BG18-like naive antibodies have a similar binding mode as BG18 iGL. Overall, these findings support the potential for both type I and type II BG18-like precursors to mature into BG18-like bnAbs (bnAbs with a BG18-like binding mode) under an appropriate sequential vaccination regimen. Given that type I and type II BG18-like precursors are enriched among epitope-specific human naive B cells, and have affinities that may confer competitive fitness in GCs, the data indicate N332-GT Env trimers are strong candidates for priming BG18-like precursors for potential maturation into HIV bnAbs in humans.

\section{Application to Vaccine Design for Pathogens Other than HIV}

We explored whether our approach to target and prime a diverse pool of antibody precursors may have applicability to other pathogens. To evaluate whether our method of germlinetargeting vaccine design could be applied beyond HIV, we carried out sequence and structural analyses for selected bnAb-antigen complexes for several major pathogens. In this non-exhaustive survey, we identified 11 potential antibody targets from five major pathogens, including hepatitis $\mathrm{C}$ virus (HCV), influenza virus, malaria, and dengue and Zika viruses (Fig. S18). According to our sequence and buried surface area analyses, these antibodies all share the ability to make a series of important contacts with antigens through templated portions of their HCDR3s (portions encoded by D or J genes), which can be targeted by vaccine design. Most of the antibodies we identified are strongly HCDR3dependent, based on a criterion of HCDR3 contributing $>30 \%$ of all surface area buried on the antibody. The strong HCDR3-dependence of the antibodies may allow for the development of related antibodies utilizing alternate $\mathrm{VH}$ or VL genes (as occurred with BG18) and hence may be advantageous for precursor frequency. With the exception of the dengue/Zika antibody EDE2 A11, all target antibodies have relatively common HCDR3 lengths of $\_22$ amino acids represented by $\geq 2 \%$ of human antibodies (30), suggesting that 
HCDR3 length will not pose a limitation on precursor frequency. All target antibodies also have mutation levels in VH and VL that are present in $\geq 1-2 \%$ of human memory B cells (30), and all but two (9/11) lack indels, thus mutation level and indels should not pose a limitation on production of similar antibodies if appropriate precursors can be primed. In some cases, the native antigen has been shown to bind to an inferred-germline or unmutated common ancestor of the target antibody (31-33), raising the question of whether a germlinetargeting approach would be necessary. We propose that even in such cases, our strategy may improve the design or validation of a vaccine priming candidate. Identification of a diverse set of antibody potential precursors with diverse HCDR3 junctions should allow for testing the breadth of precursor reactivity of the native antigen, and our design and validation strategies may optimize and/or verify breadth.

\section{Concluding Remarks}

Most antibodies, and most HIV bnAbs, recognize their target in a strongly HCDR3dependent manner. A central challenge of germline-targeting vaccine design is the large paratope sequence space and structural complexity possible for any set of antibodies targeting a conserved epitope via a shared HCDR3-dependent binding modality. Here we demonstrate the successful design of a germline-targeting immunogen for this general class of antibody recognition. We used the human repertoire and structural features of bnAb-Env binding as guides to identify a pool of potential bnAb precursors and then design an immunogen with affinity for a representative set of those precursors. This procedure was validated by the isolation of three type I BG18-like precursors from naive human B cells with N332-GT trimers and the demonstration that N332-GT nanoparticles drove a robust BG18-class B cell response in an animal model with rare BG18 precursors. Furthermore, N332-GT trimer-sorted human naive B cells were also enriched for type II BG18-like BCRs, and such precursors exhibited a BG18-like binding mode, indicating that the pool of potential BG18-like human naive precursors is larger and more diverse than originally expected. This manuscript does not demonstrate the induction of neutralizing antibodies to wild-type HIV isolates; the goal for germline-targeting priming immunogens is not to induce bnAbs directly, but rather to induce bnAb-precursor $\mathrm{B}$ cell responses that have potential to mature into bnAbs. Induction of bnAbs is the aim for a complete germline-targeting vaccine regimen, which would include a germline-targeting prime and a series of shepherding and polishing immunogens. Overall, the data presented here demonstrate a new approach to defining bnAb precursors for an epitope of interest and designing vaccine priming immunogens that take advantage of that information. This approach lays out a generalizable pathway for the development and pre-clinical validation of germline-targeting immunogens for HCDR3-dependent antibody responses.

\section{Supplementary Material}

Refer to Web version on PubMed Central for supplementary material.

\section{Acknowledgments.}

We thank H. Gristick and P. Bjorkman for providing atomic coordinates of unliganded BG18 Fab in advance of publication (17). 
Funding: This work was supported by National Institute of Allergy and Infectious Diseases (NIAID) UM1 A1100663 (Scripps Center for HIV/AIDS Vaccine Immunology and Immunogen Discovery) and UM1 AI144462 (Scripps Consortium for HIV/AIDS Vaccine Development) (to W.R.S., F.D.B., S.C., A.B.W., D.R.B) and NIAID R01 AI1 13867 (to W.R.S.); by the Ragon Institute of MGH, MIT, and Harvard (to F.D.B., W.R.S. and D.R.B.); and by the International AIDS Vaccine Initiative (IAVI) Neutralizing Antibody Consortium (NAC) and Center (to W.R.S., A.B.W., I.A.W., and D.R.B.); and through the Collaboration for AIDS Vaccine Discovery funding for the IAVI NAC Center (to W.R.S., A.B.W., I.A.W., and D.R.B.).

\section{References and Notes:}

1. Fauci AS, An HIV Vaccine Is Essential for Ending the HIV/AIDS Pandemic. JAMA 318, 15351536 (2017). [PubMed: 29052689]

2. Plotkin SA, Correlates of protection induced by vaccination. Clin Vaccine Immunol 17, 1055-1065 (2010). [PubMed: 20463105]

3. Burton DR, Poignard P, Stanfield RL, Wilson IA, Broadly neutralizing antibodies present new prospects to counter highly antigenically diverse viruses. Science 337, 183-186 (2012). [PubMed: 22798606]

4. Rappuoli R, Bottomley MJ, D'Oro U, Finco O, De Gregorio E, Reverse vaccinology 2.0: Human immunology instructs vaccine antigen design. J Exp Med 213, 469-481 (2016). [PubMed: 27022144]

5. Andrabi R, Bhiman JN, Burton DR, Strategies for a multi-stage neutralizing antibody-based HIV vaccine. Curr Opin Immunol 53, 143-151 (2018). [PubMed: 29775847]

6. Jardine $\mathrm{J}$ et al., Rational HIV immunogen design to target specific germline B cell receptors. Science 340, 711-716 (2013). [PubMed: 23539181]

7. McGuire AT et al., Engineering HIV envelope protein to activate germline B cell receptors of broadly neutralizing anti-CD4 binding site antibodies. J Exp Med 210, 655-663 (2013). [PubMed: 23530120]

8. Jardine JG et al., HIV-1 VACCINES. Priming a broadly neutralizing antibody response to HIV-1 using a germline-targeting immunogen. Science 349, 156-161 (2015). [PubMed: 26089355]

9. Jardine JG et al., HIV-1 broadly neutralizing antibody precursor B cells revealed by germlinetargeting immunogen. Science 351, 1458-1463 (2016). [PubMed: 27013733]

10. McGuire AT et al., Specifically modified Env immunogens activate B-cell precursors of broadly neutralizing HIV-1 antibodies in transgenic mice. Nat Commun 7, 10618 (2016). [PubMed: 26907590]

11. Briney B et al., Tailored Immunogens Direct Affinity Maturation toward HIV Neutralizing Antibodies. Cell 166, 1459-1470 e1411 (2016). [PubMed: 27610570]

12. Tian $\mathrm{M}$ et al., Induction of HIV Neutralizing Antibody Lineages in Mice with Diverse Precursor Repertoires. Cell 166, 1471-1484 e1418 (2016). [PubMed: 27610571]

13. Sok D et al., Priming HIV-1 broadly neutralizing antibody precursors in human Ig loci transgenic mice. Science 353, 1557-1560 (2016). [PubMed: 27608668]

14. Medina-Ramirez M et al., Design and crystal structure of a native-like HIV-1 envelope trimer that engages multiple broadly neutralizing antibody precursors in vivo. J Exp Med 214, 2573-2590 (2017). [PubMed: 28847869]

15. Havenar-Daughton $\mathrm{C}$ et al., The human naive $\mathrm{B}$ cell repertoire contains distinct subclasses for a germline-targeting HIV-1 vaccine immunogen. Sci Transl Med 10, (2018).

16. https://clinicaltrials.gov/ct2/show/NCT03547245.

17. Freund NT et al., Coexistence of potent HIV-1 broadly neutralizing antibodies and antibodysensitive viruses in a viremic controller. Sci Transl Med 9, (2017).

18. Barnes CO et al., Structural characterization of a highly-potent V3-glycan broadly neutralizing antibody bound to natively-glycosylated HIV-1 envelope. Nat Commun 9, 1251 (2018). [PubMed: 29593217]

19. Sok D, Burton DR, Recent progress in broadly neutralizing antibodies to HIV. Nat Immunol 19, 1179-1188 (2018). [PubMed: 30333615]

20. Materials and methods are available as supplementary materials at the Science website. 
21. Briney B, Inderbitzin A, Joyce C, Burton DR, Commonality despite exceptional diversity in the baseline human antibody repertoire. Nature 566, 393-397 (2019). [PubMed: 30664748]

22. Steichen JM et al., HIV Vaccine Design to Target Germline Precursors of Glycan-Dependent Broadly Neutralizing Antibodies. Immunity 45, $483-496$ (2016). [PubMed: 27617678]

23. Kulp DW et al., Structure-based design of native-like HIV-1 envelope trimers to silence nonneutralizing epitopes and eliminate CD4 binding. Nat Commun 8, 1655 (2017). [PubMed: 29162799]

24. Abbott RK et al., Precursor Frequency and Affinity Determine B Cell Competitive Fitness in Germinal Centers, Tested with Germline-Targeting HIV Vaccine Immunogens. Immunity 48, 133 146 e136 (2018). [PubMed: 29287996]

25. Lin YC et al., One-step CRISPR/Cas9 method for the rapid generation of human antibody heavy chain knock-in mice. EMBO J 37, (2018).

26. Sliepen K et al., Presenting native-like HIV-1 envelope trimers on ferritin nanoparticles improves their immunogenicity. Retrovirology 12, 82 (2015). [PubMed: 26410741]

27. Tokatlian $\mathrm{T}$ et al., Innate immune recognition of glycans targets HIV nanoparticle immunogens to germinal centers. Science 363, 649-654 (2019). [PubMed: 30573546]

28. Escolano A et al., Sequential Immunization Elicits Broadly Neutralizing Anti-HIV-1 Antibodies in Ig Knockin Mice. Cell 166, 1445-1458 e1412 (2016). [PubMed: 27610569]

29. Havenar-Daughton C, Abbott RK, Schief WR, Crotty S, When designing vaccines, consider the starting material: the human B cell repertoire. Curr Opin Immunol 53, 209-216 (2018). [PubMed: 30190230]

30. Jardine JG et al., Minimally Mutated HIV-1 Broadly Neutralizing Antibodies to Guide Reductionist Vaccine Design. PLoS pathogens 12, e1005815 (2016). [PubMed: 27560183]

31. Bangaru S et al., A Site of Vulnerability on the Influenza Virus Hemagglutinin Head Domain Trimer Interface. Cell 177, 1136-1152 e1118 (2019). [PubMed: 31100268]

32. Corti D et al., A neutralizing antibody selected from plasma cells that binds to group 1 and group 2 influenza A hemagglutinins. Science 333, 850-856 (2011). [PubMed: 21798894]

33. Flyak AI et al., HCV Broadly Neutralizing Antibodies Use a CDRH3 Disulfide Motif to Recognize an E2 Glycoprotein Site that Can Be Targeted for Vaccine Design. Cell Host Microbe 24, 703-716 e703 (2018). [PubMed: 30439340]

34. Landais E et al., Broadly Neutralizing Antibody Responses in a Large Longitudinal Sub-Saharan HIV Primary Infection Cohort. PLoS pathogens 12, e1005369 (2016). [PubMed: 26766578]

35. Suloway $\mathrm{C}$ et al., Automated molecular microscopy: the new Leginon system. Journal of structural biology 151, 41-60 (2005). [PubMed: 15890530]

36. Zheng SQ et al., MotionCor2: anisotropic correction of beam-induced motion for improved cryoelectron microscopy. Nat Methods 14, 331-332 (2017). [PubMed: 28250466]

37. Zhang K, Gctf: Real-time CTF determination and correction. Journal of structural biology 193, 112 (2016). [PubMed: 26592709]

38. Voss NR, Yoshioka CK, Radermacher M, Potter CS, Carragher B, DoG Picker and TiltPicker: software tools to facilitate particle selection in single particle electron microscopy. Journal of structural biology 166, 205-213 (2009). [PubMed: 19374019]

39. Kimanius D, Forsberg BO, Scheres SH, Lindahl E, Accelerated cryo-EM structure determination with parallelisation using GPUs in RELION-2. Elife 5, (2016).

40. Emsley P, Lohkamp B, Scott WG, Cowtan K, Features and development of Coot. Acta Crystallogr D Biol Crystallogr 66, 486-501 (2010). [PubMed: 20383002]

41. Adams PD et al., PHENIX: a comprehensive Python-based system for macromolecular structure solution. Acta Crystallogr D Biol Crystallogr 66, 213-221 (2010). [PubMed: 20124702]

42. Conway P, Tyka MD, DiMaio F, Konerding DE, Baker D, Relaxation of backbone bond geometry improves protein energy landscape modeling. Protein Sci 23, 47-55 (2014). [PubMed: 24265211]

43. Lutteke T, Frank M, von der Lieth CW, Carbohydrate Structure Suite (CSS): analysis of carbohydrate 3D structures derived from the PDB. Nucleic Acids Res 33, D242-246 (2005).

[PubMed: 15608187] 
44. Winn MD et al., Overview of the CCP4 suite and current developments. Acta Crystallogr D Biol Crystallogr 67, 235-242 (2011). [PubMed: 21460441]

45. Barad BA et al., EMRinger: side chain-directed model and map validation for 3D cryo-electron microscopy. Nat Methods 12, 943-946 (2015). [PubMed: 26280328]

46. Chen VB et al., MolProbity: all-atom structure validation for macromolecular crystallography. Acta Crystallogr D Biol Crystallogr 66, 12-21 (2010). [PubMed: 20057044]

47. Pettersen EF et al., UCSF Chimera--a visualization system for exploratory research and analysis. Journal of computational chemistry 25, 1605-1612 (2004). [PubMed: 15264254]

48. Willis JR et al., Redesigned HIV antibodies exhibit enhanced neutralizing potency and breadth. J Clin Invest 125, 2523-2531 (2015). [PubMed: 25985274]

49. DeKosky BJ et al., In-depth determination and analysis of the human paired heavy- and light-chain antibody repertoire. Nat Med 21, 86-91 (2015). [PubMed: 25501908]

50. Lefranc MP et al., IMGT(R), the international ImMunoGeneTics information system(R) 25 years on. Nucleic Acids Res 43, D413-422 (2015). [PubMed: 25378316]

51. Krissinel E, Henrick K, Inference of macromolecular assemblies from crystalline state. J Mol Biol 372, 774-797 (2007). [PubMed: 17681537]

52. Lefranc MP et al., IMGT unique numbering for immunoglobulin and T cell receptor variable domains and Ig superfamily V-like domains. Dev Comp Immunol 27, 55-77 (2003). [PubMed: 12477501]

53. Julien JP et al., Broadly neutralizing antibody PGT121 allosterically modulates CD4 binding via recognition of the HIV-1 gp120 V3 base and multiple surrounding glycans. PLoS pathogens 9, e1003342 (2013). [PubMed: 23658524]

54. Julien JP et al., Crystal structure of a soluble cleaved HIV-1 envelope trimer. Science 342, 14771483 (2013). [PubMed: 24179159]

55. Dosenovic P et al., Anti-HIV-1 B cell responses are dependent on B cell precursor frequency and antigen-binding affinity. Proceedings of the National Academy of Sciences of the United States of America 115, 4743-4748 (2018). [PubMed: 29666227]

56. Dreyfus C et al., Highly conserved protective epitopes on influenza B viruses. Science 337, $1343-$ 1348 (2012). [PubMed: 22878502]

57. Lang S et al., Antibody 27F3 Broadly Targets Influenza A Group 1 and 2 Hemagglutinins through a Further Variation in VH1-69 Antibody Orientation on the HA Stem. Cell Rep 20, 2935-2943 (2017). [PubMed: 28930686]

58. Joyce MG et al., Vaccine-Induced Antibodies that Neutralize Group 1 and Group 2 Influenza A Viruses. Cell 166, 609-623 (2016). [PubMed: 27453470]

59. Oyen D et al., Structural basis for antibody recognition of the NANP repeats in Plasmodium falciparum circumsporozoite protein. Proceedings of the National Academy of Sciences of the United States of America 114, E10438-E10445 (2017). [PubMed: 29138320]

60. Kisalu NK et al., A human monoclonal antibody prevents malaria infection by targeting a new site of vulnerability on the parasite. Nat Med 24, 408-416 (2018). [PubMed: 29554083]

61. Rouvinski A et al., Recognition determinants of broadly neutralizing human antibodies against dengue viruses. Nature 520, 109-113 (2015). [PubMed: 25581790]

62. Dejnirattisai W et al., A new class of highly potent, broadly neutralizing antibodies isolated from viremic patients infected with dengue virus. Nat Immunol 16, 170-177 (2015). [PubMed: 25501631] 
A

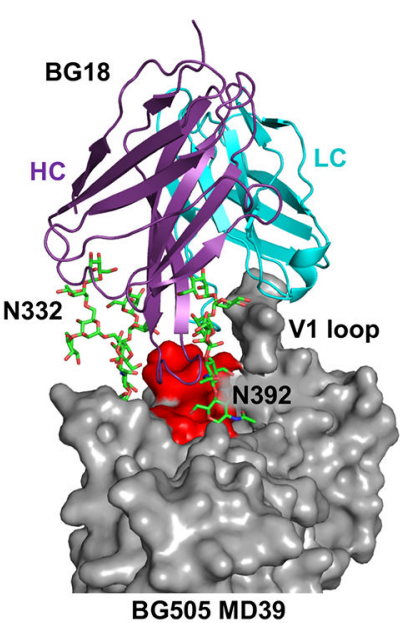

C

Selection Ab Immunogen Library

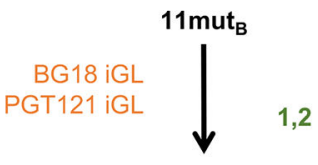

17 mut $_{\mathrm{E}}$

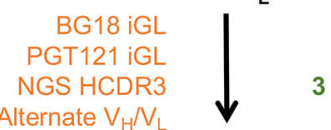

N332-GT1
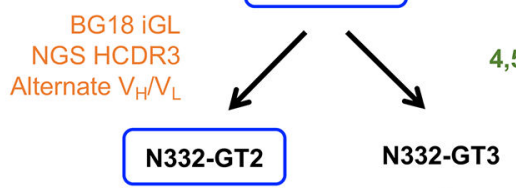

N332-GT2

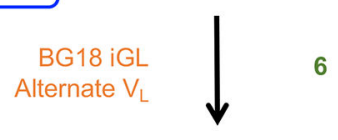

N332-GT4

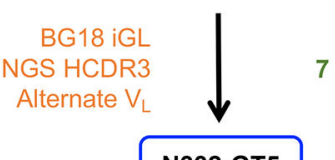

N332-GT5
B

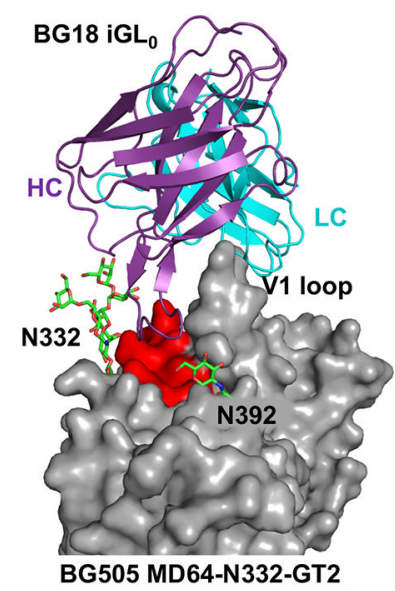

D
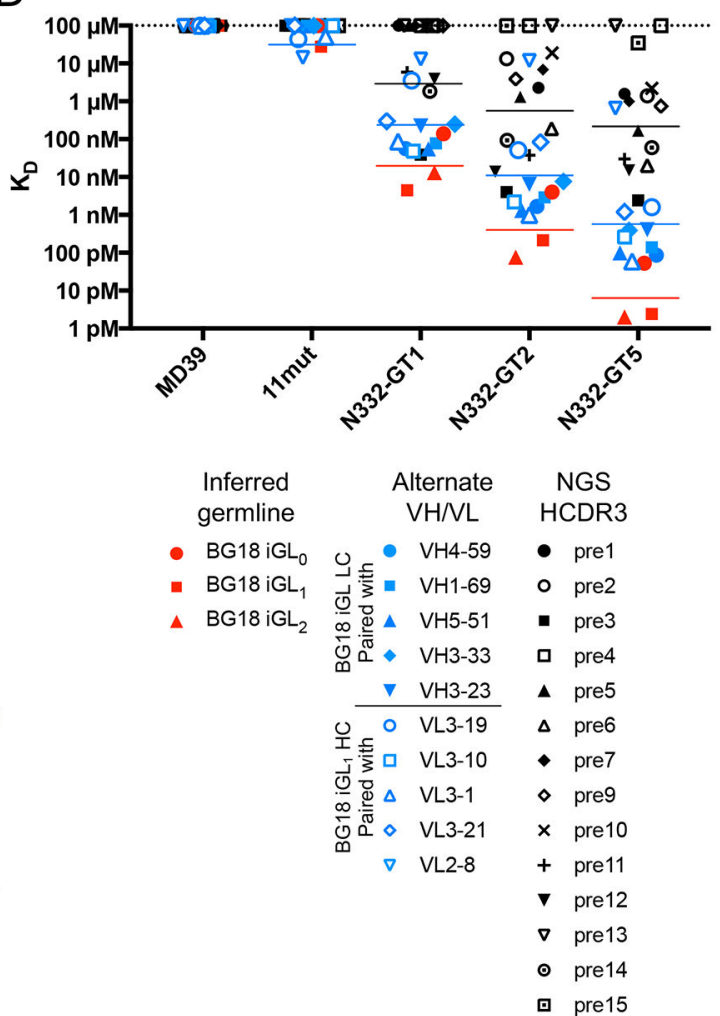

Fig. 1. Engineering germline-targeting trimers for an HCDR3-dependent bnAb.

(A) Cryo-EM structure of BG18 (HC, purple; LC, cyan) bound to BG505 MD39 Env trimer (gray, with N332 and N392 glycans shown as green sticks), and conserved residues near the base of V3 [G324, D325, I326, R327, Q328, A329, H330, T415, L416 and P417] colored red). (B) Cryo-EM structure of BG18 $\mathrm{iGL}_{0}$ in complex with N332-GT2 Env trimer with MD64 stabilizing mutations (23); coloring as in (A). (C) Schematic of the directed evolution process to design N332-GT1, -GT2 and -GT5. (D) N332-GT binding affinities $\left(\mathrm{K}_{\mathrm{D}}\right)$ for BG18 iGL 0 -2 (red), BG18 iGL 1 with alternate germline $\mathrm{V}_{\mathrm{L}}$ (blue open symbols) or $\mathrm{V}_{\mathrm{H}}$ genes (blue filled symbols), and BG18 iGL containing NGS-derived HCDR3s (pre1-15) 
(black). MD39 is the reference Env trimer with no germline-targeting mutations. Pre8 was found to be highly polyreactive and was not included in the analysis. Solid black, blue, and red lines indicate the geomean $\mathrm{K}_{\mathrm{D}} \mathrm{s}$ for NGS-derived precursors, alternate $\mathrm{V}_{\mathrm{H}} / \mathrm{V}_{\mathrm{L}}$ precursors, and inferred germline precursors, respectively. Dashed line indicates the limit of detection. 
A

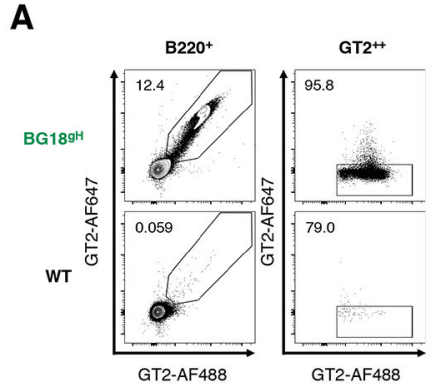

D

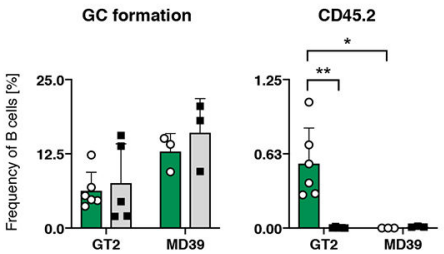

G

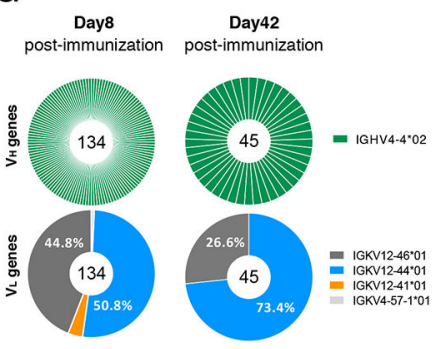

J

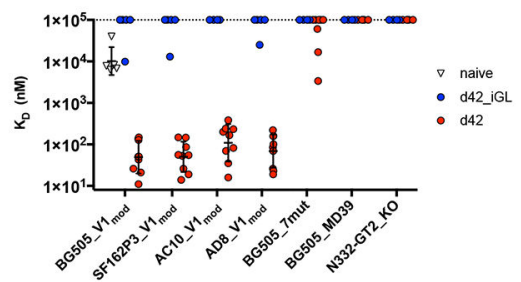

B

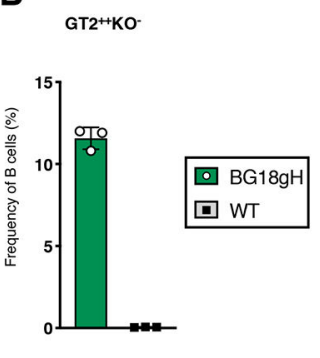

E

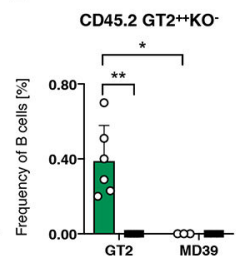

H

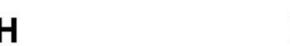

I

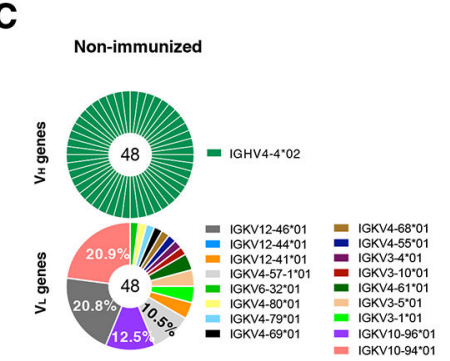

F
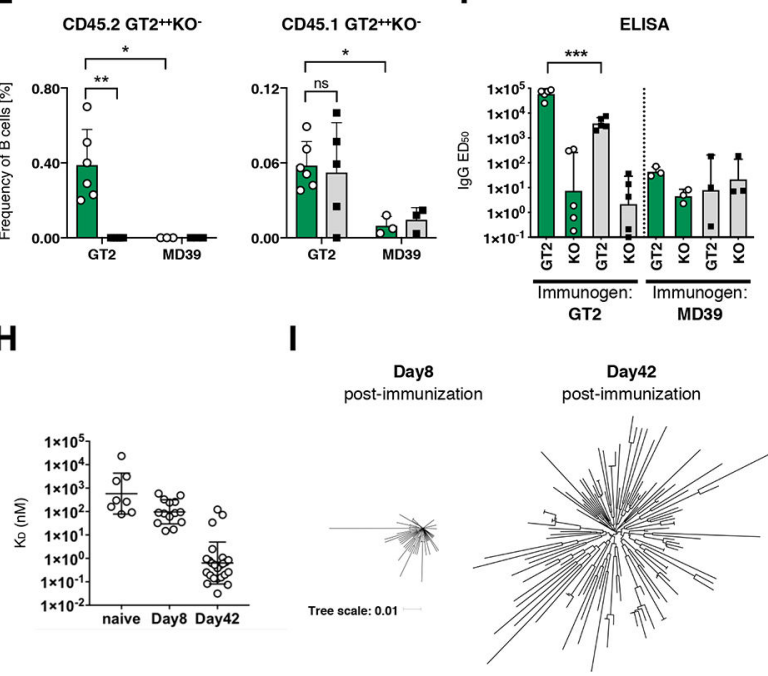

K

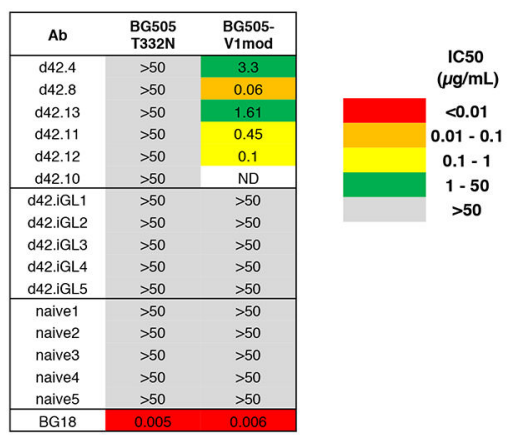

Fig. 2. Immunization of $\mathrm{BG}^{\mathrm{gH}} \mathrm{B}$ cell adoptive transfer recipient mice with N332-GT2 Env nanoparticles.

(A) Gating strategy to identify epitope-specific (N332-GT2 ${ }^{++} / \mathrm{N} 332-\mathrm{GT} 2-\mathrm{KO}^{-}$) B cells in $\mathrm{BG} 18^{\mathrm{gH}}$ and WT mice. Each symbol represents a different mouse. Bars indicate mean $\pm \mathrm{SD}$ from experiments in 3 mice in each model. (B) Frequency of epitope-specific B cells in nonimmunized BG18 ${ }^{\mathrm{gH}}$ and WT mice. (C) Distribution of $\mathrm{V}_{\mathrm{H}}$ and $\mathrm{V}_{\mathrm{L}}$ genes in epitope-specific naive $\mathrm{B}$ cells in non-immunized BG18 ${ }^{\mathrm{gH}}$ mice. (D) Frequency of GC B cells (left) or CD45.2 ${ }^{+}$GC B cells (right) in four immunization conditions. Each symbol represents a different mouse. Bars indicate mean $\pm \mathrm{SD}$ from experiments in the following number of mice in each condition: BG18gH (GT2), $\mathrm{n}=6$; WT (GT2), $\mathrm{n}=5$; BG18gH (MD39), $\mathrm{n}=3$; WT (MD39), $n=3$. (E) Frequency of CD45.2 $2^{+}$(left) or CD45.1 $1^{+}$(right) epitope-specific B cells in four immunization conditions. Each symbol represents a different mouse. Bars 
indicate mean $\pm \mathrm{SD}$ from experiments in the following number of mice in each condition: BG18gH (GT2), $\mathrm{n}=6$; WT (GT2), $\mathrm{n}=5$; BG18gH (MD39), $\mathrm{n}=3$; WT (MD39), $\mathrm{n}=3$. (F) Serum ELISA 50\% equilibrium dilution values for N332-GT2 and N332-GT2-KO at day 14 after immunization for four immunization conditions. Each symbol represents a different mouse. Bars indicate geometric mean and geometric SD from experiments in the following number of mice in each condition: BG18gH (GT2), $\mathrm{n}=5$; WT (GT2), $\mathrm{n}=5$; $\mathrm{BG18 \textrm {gH }}$ (MD39), $\mathrm{n}=3$; WT (MD39), $\mathrm{n}=3$. Student's t-test; $\mathrm{ns}, \mathrm{P}>0.05 ; *, \mathrm{P}<0.05 ; * *, \mathrm{P}<0.01$. Data in $(\mathrm{A})-(\mathrm{F})$ are from one of three representative experiments with three or more animals in each group. (G) Distribution of $\mathrm{V}_{\mathrm{H}}$ and $\mathrm{V}_{\mathrm{L}}$ genes in epitope-specific GC $\left(\mathrm{CD} 38^{\text {low }} \mathrm{CD}^{2} 5^{+}\right) \mathrm{B}$ cells 8 and 42 days after immunization of $\mathrm{BG} 18^{\mathrm{gH}} \mathrm{B}$ cell adoptive transfer recipient mice. $(\mathrm{H})$ SPR dissociation constants $\left(\mathrm{K}_{\mathrm{D}}\right)$ for N332-GT2 trimer binding to epitope-specific Fabs derived from naive B cells in non-immunized BG1 $8^{\mathrm{gH}}$ mice and GC B cells 8 and 42 days after immunization of $\mathrm{BG} 18^{\mathrm{gH}} \mathrm{B}$ cell adoptive transfer recipient mice. Each symbol corresponds to a different Fab and represents one or two measurements. Bars indicate geometric mean and geometric SD. (I) Phylogenetic trees of BCR HCs isolated from epitope-specific CD45.2 ${ }^{+}$B cells 8 and 42 days after immunization with N332-GT2 NPs. Tree scale indicates the number of substitutions per site. (J) SPR dissociation constants $\left(\mathrm{K}_{\mathrm{D}}\right)$ for the five highest affinity naive Fabs from $(\mathrm{H})$ binding to the V1 loop-modified BG505 trimer (BG505_V1 mod), and for nine of the high affinity day 42 Fabs from $(\mathrm{H})$ and five inferred-germline variants of the high-affinity day 42 Fabs (Day42.iGL) binding to V1 loop-modified trimers from BG505 and three other HIV isolates (SF162P3, AC10, AD8) as well as a BG505 trimer with a less modified V1 loop (BG505_7mut), a native-like trimer (BG505_MD39) and an epitope-KO trimer (N332-GT2_KO). Each symbol corresponds to a different $\mathrm{Fab}$ and represents one or two measurements. Bars indicate geometric mean and geometric SD. Dashed line indicates limit of detection. (K) Neutralization potency (IC50) against native (BG505 T332N) and V1 loop-modified (BG505-V1mod) pseudoviruses, for the BG18 bnAb, the five highest affinity naive Fabs from $(\mathrm{H})$, five inferred-germline variants of the high-affinity day 42 Fabs (Day42.iGL), and five high affinity day 42 Fabs. Each IC50 is an average from two measurements. ND indicates not determined. 
A
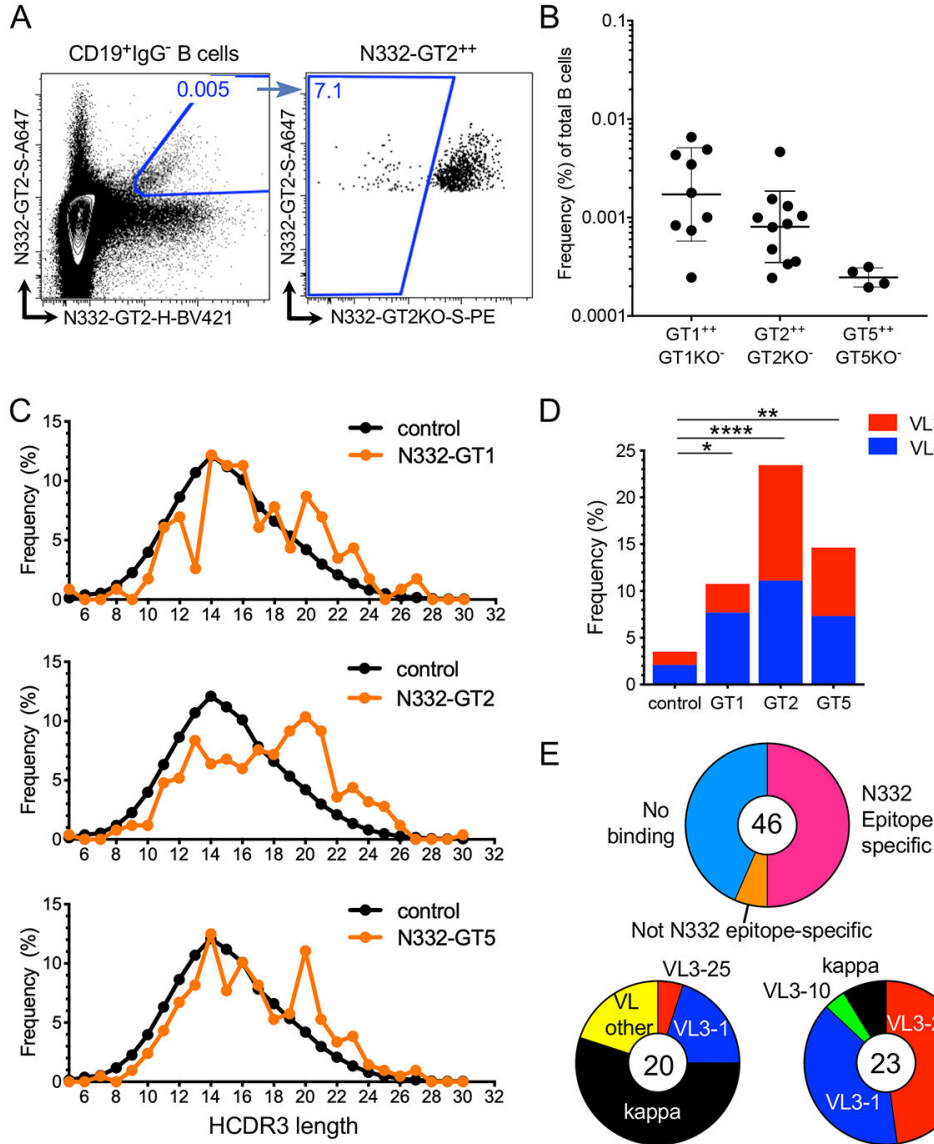

F

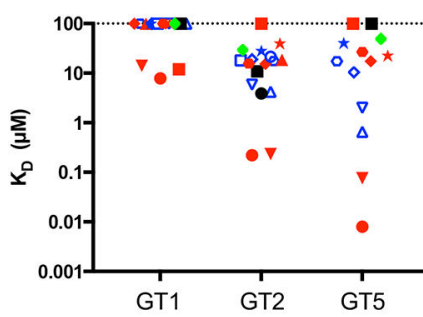

D

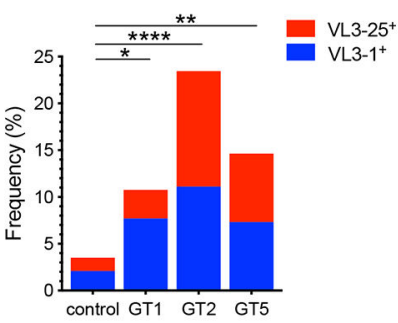

E
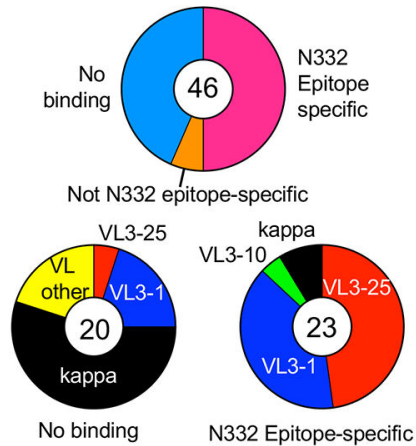

$\underline{\mathrm{VL3}^{2} 5^{+}} \quad \underline{\mathrm{VL3}-1^{+}} \quad \underline{\mathrm{Kappa}^{+}}$

- HMP1 ○ HMP8 $\overline{\text { HMP19 }}$

- HMP10 a HMP11 — HMP49

$\nabla$ HMP42 $\nabla$ HMP43 VL3-10+

- HMP58 $\bullet$ HMP44 $\frac{\text { HMP65 }}{\text { HMP6 }}$

- hMP60 ○ hMP57

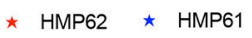

\ HMP26 $\triangle$ HMP30

Fig. 3. Naive human B cells sorted with N332-GT Env trimers.

(A) Gating strategy for N332-GT epitope-specific sorting of naive human B cells. (B) Frequency of epitope-specific B cells among IgG-negative B cells. Each symbol represents a different human subject. Bars indicate geometric mean and geometric mean SD from the following number of independent subjects: N332-GT1, $n=9$; N332-GT2, $n=11$; N332$\mathrm{GT} 5, \mathrm{n}=4$. (C) HCDR3 length distribution from epitope-specific sorted cells compared to control B cells. (D) Frequency of $\mathrm{V}_{\mathrm{L}} 3-25$ or $\mathrm{V}_{\mathrm{L}}$ 3-1 LCs from epitope-specific sorted cells relative to control B cells. Significance of differences from control was evaluated by a Chisqaured test. *, $\mathrm{P}=0.01 ; * *, \mathrm{P}=0.005 ; * * * *, \mathrm{P}=0.0001$. (E) SPR-derived binding specificities for 46 HMP Fabs corresponding to epitope-specific naive human B cells isolated by N332GT1 or N332-GT2 (top), with light chain V gene usage for non-binding Fabs (bottom left) and for N332-epitope-specific Fabs (bottom right). (F) SPR dissociation constants for HMP 
epitope-specific Fabs isolated with N332-GT1 and N332-GT2 Env trimers. Dashed line indicates the limit of detection. 
A

\begin{tabular}{lcccccccc} 
Name & $\begin{array}{c}\text { Isolated } \\
\text { with }\end{array}$ & \multicolumn{1}{c}{ HCDR3 } & Length & VH & D & JH & VL & JL \\
\hline BG18-mat & - & ARNAIRIYGVVALGEWFHYGMDV & 23 & $4-4$ & $3-3$ & 6 & L3-25 & 2 or 3 \\
BG18-iGL & - & ARNAITIFGVVIIGEYYYYGMDV & 23 & $4-4$ & $3-3$ & 6 & L3-25 & 2 or 3 \\
\hline HMP1 & GT1 & AREGFTIFGVVTFSEGYYYGMDV & 23 & $4-61$ & $3-3$ & 6 & L3-25 & 2 or 3 \\
HMP68 & GT5 & TRAPITIFGVVLLDYYYYYMDV & 23 & $3-49$ & $3-3$ & 6 & ND & ND \\
HMP69 & GT5 & ARDRITIFGVVIIGENYYYGMDV & 23 & $3-11$ & $3-3$ & 6 & L3-25 & 2 or 3
\end{tabular}

\begin{tabular}{|c|c|c|c|c|c|c|c|c|}
\hline Name & Isolated with & HCDR3 & Length & VH & D & JH & VL & $\mathrm{JL}$ \\
\hline HMP30 & GT2 & AKDFLLSTSGYLSYGDYYYYGMDV & 24 & 3-30 & $5-18$ & 6 & L3-1 & 2 or 3 \\
\hline HMP70 & GT5 & ARSWALFCSSTSCTNYYYYYGMDV & 24 & $1-8$ & $2-2$ & 6 & L3-25 & 3 \\
\hline HMP57 & GT2 & TTDIGYGDYLRSLDYYYYYGMDV & 23 & $3-15$ & 4-17 & 6 & L3-1 & 2 or 3 \\
\hline HMP65 & GT2 & ARDQIREEWELLPEYYYYGMDV & 22 & $3-30-3$ & $1-26$ & 6 & L3-10 & 3 \\
\hline HMP71 & GT5 & AREESIVLMVYAIPADKDAFDI & 22 & $1-2$ & $2-8$ & 3 & L3-1 & 2 or 3 \\
\hline HMP72 & GT5 & ARKGPEGDGYNWELGTLYYFDY & 22 & $4-59$ & $5-24$ & 4 & L3-25 & 3 \\
\hline HMP8 & GT1 & AROKVDYDFWSGYPAGYGMDV & 21 & $3-74$ & $3-3$ & 6 & L3-1 & 2 or 3 \\
\hline HMP26 & GT2 & ARDPTPNYYDSSGYWVGAFDI & 21 & 4-59 & 3-22 & 3 & L3-25 & 2 or 3 \\
\hline HMP42 & GT2 & ARDRGREWELESYYYYYYMMVV & 21 & $1-2$ & $1-26$ & 6 & L3-25 & 2 or 3 \\
\hline HMP43 & GT2 & AKDIESRYFDWDNYYYYGMDV & 21 & 3-9 & $3-9$ & 6 & L3-1 & 2 or 3 \\
\hline HMP44 & GT2 & ARDVSRNWGWEEDYYYYGMDV & 21 & $3-30-3$ & 2-21 & 6 & L3-1 & 2 or 3 \\
\hline HMP58 & GT2 & ARVKYSSSWYVDDYYYGMDV & 20 & $3-74$ & $6-13$ & 6 & L3-25 & 2 or 3 \\
\hline HMP60 & GT2 & ARGDLYYYDSSGYYYYGMDV & 20 & 3-30 & 3-22 & 6 & L3-25 & 7 \\
\hline HMP61 & GT2 & ARAWDYDYVWGSYGVDGMDV & 20 & $4-34$ & 3-16 & 6 & L3-1 & 2 or 3 \\
\hline HMP35 & GT2 & AKGPOHYYDSSGYPEYYFDY & 20 & $3-23$ & 3-22 & 4 & L3-25 & 2 or 3 \\
\hline HMP38 & GT1 & ARDGTLYYYDSSGYYSYFDY & 20 & $3-30-3$ & 3-22 & 4 & L3-1 & 2 or 3 \\
\hline HMP39 & GT2 & ARDRDSYYYDSSGYSDYFDY & 20 & 3-33 & 3-22 & 4 & L3-25 & 2 or 3 \\
\hline HMP40 & GT2 & ARGSVYYYDSSGYFWDAFDI & 20 & $3-30-3$ & 3-22 & 3 & L3-1 & 1 \\
\hline HMP59 & GT2 & ARDATMSIAPNYYYYYGMDV & 20 & $3-33$ & $6-6$ & 6 & L3-25 & 2 or 3 \\
\hline HMP73 & GT5 & AREWDGGYSGYDSGDWYFDL & 20 & 3-48 & $5-12$ & 2 & L3-1 & 2 or 3 \\
\hline
\end{tabular}

C

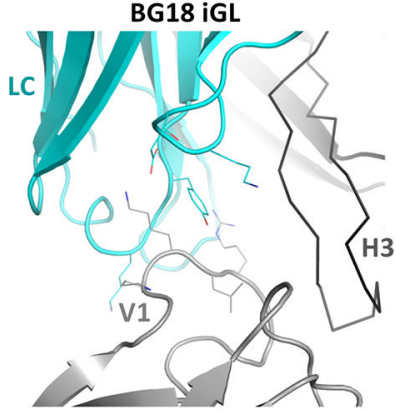

N332-GT2

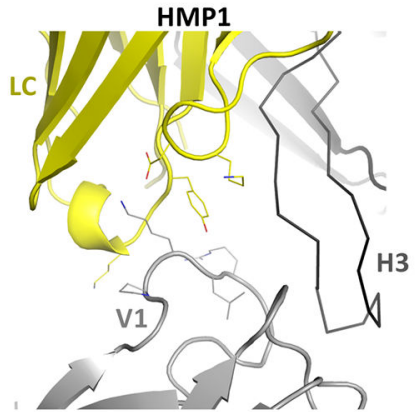

N332-GT5

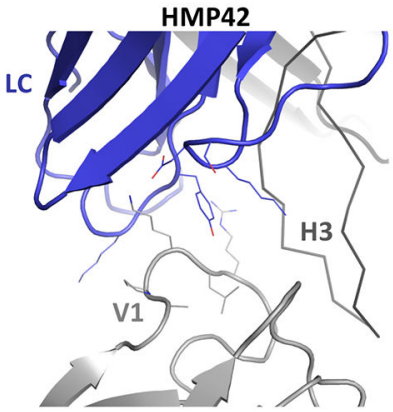

N332-GT5

D

BG18

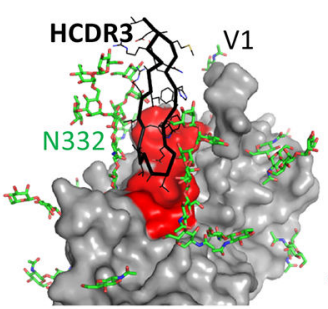

MD39
BG18 iGL

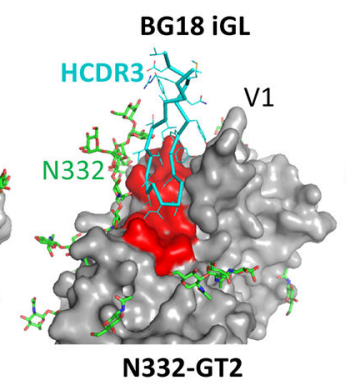

N332-GT2

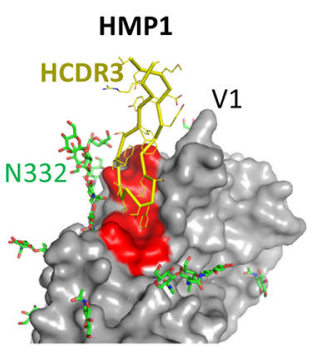

N332-GT5

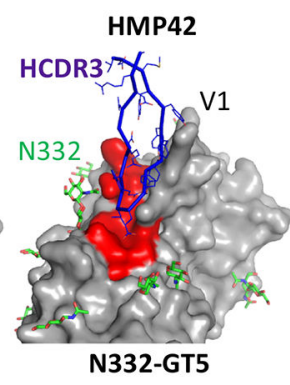

Fig. 4. Sequence and structural characterization of type I and type II BG18-like naive antibodies isolated by N332-GT Env trimers.

(A) HCDR3 sequence and gene segment assignments for three type I BG18-like naive human precursor antibodies. V, D, and J gene segments are colored blue, red, and green, respectively. (B) HCDR3 sequence and gene segment assignments for 20 type II BG18-like precursor antibodies. (C) Cryo-EM structural analysis of type I (HMP1) and type II (HMP42) precursor antibody LC interactions with N332-GT5 compared to BG18 iGL LC interactions with N332-GT2. Gp120 is colored gray, and the LCs are colored cyan, yellow, and blue for BG18 iGL, HMP1 and HMP42, respectively. (D) Cryo-EM structural analysis of type I and type II precursor HCDR3 interactions with N332-GT5 compared to HCDR3 
interactions for BG18+MD39 and BG18iGL+N332-GT2 complexes. Gp120 is colored gray with conserved residues (or the corresponding germline-targeting amino acids) near the base of V3 in red as in Fig. 1A. Glycans are shown as green sticks. 